\title{
28 Research Soure \\ Identification of a Four-miRNA Signature Predicts Prognosis in Papillary Thyroid Cancer
}

\section{YANG FAN}

Wenzhou Medical University

Hongzhong zhou ( $\nabla$ zhouhongzhongwmu@126.com )

Wenzhou Medical University

\section{Research Article}

Keywords: Papillary thyroid cancer, miRNA, prognosis, signature

Posted Date: August 2nd, 2021

DOI: https://doi.org/10.21203/rs.3.rs-743455/v1

License: (c) (1) This work is licensed under a Creative Commons Attribution 4.0 International License. Read Full License 


\section{Abstract}

Background: In newly diagnosed patients with thyroid cancer, papillary thyroid cancer (PTC), accounts for ninety percent of all cases. Although PTC is known as a relatively adolescent malignant disease, there still is a high possibility of recurrence in PTC patients, who suffered from poor prognosis. Therefore, new biomarkers are necessary to guide more effective stratification of PTC patients and personalization of therapy to avoid overtreatment or inadequate treatment. Accumulating evidence demonstrates that miRNAs have broad application prospects as diagnostic biomarkers in cancer.

Methods: The present study aims to explore novel markers consists of miRNA-associated signature for PTC prognostication, utilizing data from the TCGA database. We obtained and analyzed data of 497 PTC patients from the TCGA. The patients were randomly assigned to a training cohort or testing cohort.

Results: We discovered 237 differentially expressed miRNAs in tumorous thyroid tissues comparing to normal tissues The effect evaluation of excavated differently expressed miRNAs was conducted by our risk score model. We then successfully generated a four-miRNA potential prognostic signature, which reliably distinguishes patients from high risk and low risk with a significant difference in overall survival $(P<0.01)$ and was effective in predicting five-year disease survival.

Conclusions: Our results indicated a four miRNAs signature that has a robust predicting effect on the prognosis of PTC. Therefore, we would recommend more radical treatment and closer follow-up of highrisk individuals.

\section{Background}

Thyroid cancer is the most prevalent endocrine malignancy in the world, and its incidence is rapidly rising

${ }^{1}$. It ranks the fifth most common cancer in female patients, whose incidence is about three times higher than males in most regions and populations ${ }^{2,3}$. Differentiated thyroid cancer derives from follicular epithelial cells; the main subtypes contain of papillary thyroid cancer (PTC), follicular thyroid cancer (FTC), and Hurthle cell carcinoma ${ }^{4}$. The most common histological subtype of thyroid cancer is PTC, which accounts for $90 \%$ of newly diagnosed thyroid cancers and has the best prognosis among all subtypes $^{4}$. PTC usually behaves like an indolent disease in most patients and can be well controlled or cured by means of the appropriate surgical procedure or with the help of radio-iodine. However, the recurrence rate of PTC is still high, and the dedifferentiation of PTC would potentially lead to invasiveness and poor prognosis 5 .

Given that PTC is a heterogeneous disease, optimal treatment for PTC patients has long been a heated controversy. On the one hand, more aggressive treatment of cancer is able to reduce the recurrence of disease and mortality rates. On the other hand, it will also give rise to treatment-related complications ${ }^{6}$. To alleviate this dilemma, we need to improve risk stratification to identify patients with worse outcomes 
more accurately. Therefore, introducing new biomarkers as an advanced method in improving the overall survival (OS) of PTC patients would be favored.

MicroRNAs (miRNAs) are types of non-coding, single-stranded RNA molecules, which consist of about 18 to 25 nucleotides. The specific binding of miRNAs to the complementary mRNA can either facilitate mRNA degradation or prevent mRNA translation into protein. ${ }^{7}$. Previous reports have suggested that miRNAs were essential in the tumorigenesis and progression of $\mathrm{PTC}^{8,9}$. Accumulating evidence has also demonstrated that miRNAs had broad application prospects, such as diagnostic biomarkers and therapeutic targets in cancer ${ }^{10}$. However, the limitation of existent studies includes the inadequate sample quantitation and lack of comprehensive analysis on a large amount of PTC samples. Now with the help of The Cancer Genome Atlas (TCGA) database, we are able to investigate cancer-specific signatures, which contain large-scale miRNAs expression data and prognostic survival data.

In this study, we utilized data from the TCGA database to conduct a comprehensive analysis; therefore, screening out differentially expressed miRNAs. We then assessed the prognostic value of these miRNAs utilizing a risk score model. A panel of four miRNAs was generated as a prognostic signature, which has been tested in PTC patients. Such a practical tool has satisfying potential in the stratification of PTC patients and individualized therapy to avoid overtreatment or inadequate treatment.

\section{Methods}

Expression profiles collection

Level three miRNASeq datasets of 507 PTC and 58 normal samples, as well as corresponding clinical data of PTC patients, were extracted from The Cancer Genome Atlas (TCGA) database (http://cancergenome.nih.gov) on December 12, 2019 ${ }^{11}$. The inclusion criteria of studied samples were as shown below: 1) data that contain both miRNA sequencing and clinical information; 2) the sample had prognosis information; 3) histological typing was PTC. There were 497 PTC samples met our criteria and were selected for further analysis. The entire set was randomly separated into a training cohort (249 cases) and a test cohort (248 cases). The detailed baseline characteristics of the entire set were listed in Table S1.

Identification of differentially expressed miRNA

Analysis of global miRNA expression profile detected 2202 miRNAs. Then, the miRNA expression profiles were standardized using the $\mathrm{R}$ package of edgeR ${ }^{12}$. EdgeR was also utilized to sift out the differentially expressed miRNAs according to the following criteria: 1) fold change (FC) $>2$ for up- or down-regulation and 2) false discovery rate $(F D R)<0.05$. Based on the analysis of the differentially expressed miRNA, a volcano plot was produced with label colors that determined by the filtering criteria.

Selection of candidate Prognosis biomarkers 
Firstly, univariate cox regression analysis was used to sift out each differently expressed miRNAs that related to patients' over-survival rate (OS). Subsequently, these differently expressed miRNAs with a $P<$ 0.01 were selected into Least absolute shrinkage, and selection operator analysis (LASSO). LASSO analysis constructed a more refined model by constructing a penalty function. Finally, we established general multivariate stepwise Cox regression models to identify which of the significant miRNAs was an independent predictor of prognosis.

Construction of miRNA signature

MiRNAs that has notable association with OS in the multivariate Cox regression analysis were utilized to construct the miRNA signature, which was used to estimate the prognostic risk score for each patient. The miRNA signature was built using the coefficients obtained from the Cox regression analysis, the standard were as follows: Risk score $=(-0.001 \times$ hsa-miR-181a-2-3p $)+(0.003 \times$ hsa-miR-138-5p $)+$ $(-0.018 \times h s a-m i R-424-3 p)+(0.284 \times h s a-m i R-612)$. Subsequently, according to the same median risk score as the cutoff point, patients in the both training and testing cohorts were divided into the low-risk group and the high-risk group. Next, the area under the curve (AUC) of time-dependent receiver operating characteristic (ROC) analysis was analyzed to reveal the predictive effect of the miRNA-based classifier and prognostic model. To make the prognostic miRNAs signature more convenient in clinical practice, we also constructed a prognostic nomogram. Furthermore, a calibration curve was carried out to assess the consistency of model prediction and the actual outcome.

Functional analysis of miRNA signature

The miRDB (http://www.mirdb.org/miRDB/) and TargetScan (http://www.targetscan.org/) were used for the prediction target genes of four miRNAs. Furthermore, overlapping target genes from these two online analysis database were analyzed by the functional enrichment analysis tool FunRich ${ }^{13}$. Gene Ontology (GO) and Kyoto Encyclopedia of Genes and Genomes (KEGG) pathway enrichment analyses were used to analyze the function of these target genes. These target genes of four miRNAs were listed in Table S2.

\section{Statistical analysis}

The Mann-Whitney $\mathrm{U}$ test and the $\chi^{2}$ test was used to analyzed the associations of continuous and categorical variables between the training cohort and testing cohort, respectively. Survival analyses were compared by log-rank tests while the Kaplan-Meier method was adopted to plot the survival curves. A $P$ value $<0.05$ was considered statistically significant. All statistical analyses were performed by SPSS version 22.0 (Chicago, IL) and $\mathrm{R}$ version 3.6.1.

\section{Results}

Baseline clinical characteristics of PTC patients 
We extracted and investigated data of 497 PTC patients from the TCGA database. The patients were randomly assigned into the training cohort or testing cohort. In Table 1, we demonstrated the detailed clinical characteristics (age, sex, vital status, stages, and T/N/M classification) of both cohorts, finding no significant differences between the two groups $(P>0.05)$. 
Table 1

Clinical characteristics of 497 patients with PTC

\begin{tabular}{|c|c|c|c|}
\hline Variable & Train cohort & Test cohort & $P$ value \\
\hline & $(\mathrm{n}=249)$ & $(n=248)$ & \\
\hline Age & & & 0.968 \\
\hline$<45$ & 112 & 112 & \\
\hline$\geq 45$ & 137 & 138 & \\
\hline Sex & & & 0.064 \\
\hline Male & 75 & 57 & \\
\hline Female & 174 & 193 & \\
\hline Vital status & & & 0.617 \\
\hline Death & 9 & 7 & \\
\hline Alive & 240 & 241 & \\
\hline Stages & & & 0.768 \\
\hline प & 140 & 138 & \\
\hline प & 28 & 23 & \\
\hline प & 53 & 61 & \\
\hline प & 28 & 26 & \\
\hline T classification & & & 0.302 \\
\hline T1 & 67 & 76 & \\
\hline T2 & 92 & 74 & \\
\hline T3 & 78 & 85 & \\
\hline T4 & 12 & 11 & \\
\hline Tx & 0 & 2 & \\
\hline $\mathrm{N}$ classification & & & 0.166 \\
\hline NO & 107 & 121 & \\
\hline N1 & 120 & 99 & \\
\hline$N x$ & 22 & 28 & \\
\hline M classification & & & 0.584 \\
\hline M0 & 139 & 139 & \\
\hline
\end{tabular}




\begin{tabular}{|llll|}
\hline Variable & Train cohort & Test cohort & $\boldsymbol{P}$ value \\
\hline M1 & 3 & 6 \\
Mx & 107 & 103 \\
\hline
\end{tabular}

Selection of differentially expressed miRNAs and candidate diagnostic biomarkers

To select significantly differentially expressed miRNA in the 497 PTC tissues and 59 adjacent normal thyroid tissues (Fig. 1), we performed volcano plot to evaluate miRNA expression variation with the standard of $F C>2$ or $<0.5$, as well as FDR $<0.05$. A total of 237 miRNAs were differentially expressed in the cancerous tissues comparing to the normal tissues, including 172 up-regulated miRNAs and 65 downregulated miRNAs. These 237 significantly differently expressed miRNAs were identified as potential prognostic biomarkers for PTC. To screen out the OS-related miRNAs, univariate cox regression analysis was performed for these significantly differentially expressed miRNAs in the training cohort. Subsequently, we found five miRNAs were distinctly associated with the OS of PTC (Table 2).

Table 2

Univariate cox regression analyses five miRNA were distinctly associated with OS of PTC in train cohort

\begin{tabular}{|lllll|}
\hline miRNA & Pvalue & HR & Lower 95\%Cl & Upper 95\%Cl \\
\hline hsa-miR-138-5p & 0.0001 & 1.002 & 1.001 & 1.004 \\
\hline hsa-miR-1179 & 0.0007 & 1.012 & 1.005 & 1.019 \\
\hline hsa-miR-138-1-3p & 0.0010 & 1.028 & 1.011 & 1.045 \\
\hline hsa-miR-612 & 0.0064 & 1.372 & 1.093 & 1.722 \\
\hline hsa-miR-7-2-3p & 0.0077 & 1.011 & 1.003 & 1.019 \\
\hline Abbreviations: Cl, confidence interval; HR, hazard ratio; PTC, papillary thyroid cancer. \\
\hline
\end{tabular}

Construction of the miRNA prognostic signature

To construct the miRNA prognostic signature, these five miRNAs were further selected into LASSO analysis and multivariate cox regression analysis. The lambda value was set using the lambda.min, which is the value of lambda, giving minimum mean cross-validated error. Four miRNAs with non-zero coefficients were defined. The result of the LASSO analysis was shown in Figure S1. We finally managed to established four miRNAs which have independent prognostic effect for PTC in the training cohort based on the LASSO and Cox regression models (Table 3). To reveal the weight of each weighting coefficient of miRNAs clearly, forest figure was presented in Figure S2. We then chose the same median risk score in the two aforementioned independent cohorts as the cutoff point, classifying patients into the low-risk group and the high-risk group. Figure 2 showed the distribution of the miRNA-based risk score, 
OS, and four miRNAs expression profiles of the training cohort and testing cohort. Kaplan-Meier survival analysis indicated a much worse prognosis $(P<0.001)$ in the high-risk group (Fig. $3 \mathrm{~A})$.

Table 3

Multivariate cox regression analyses of four miRNA were distinctly associated with OS of PTC in train cohort

\begin{tabular}{|llllll|}
\hline miRNA & coefficient & $P$ value & HR & Lower 95\%Cl & Upper 95\%Cl \\
\hline hsa-miR-181a-2-3p & -0.001 & 0.0800 & 1.000 & 1.000 & 1.000 \\
\hline hsa-miR-138-5p & 0.003 & 0.0001 & 1.003 & 1.001 & 1.004 \\
\hline hsa-miR-424-3p & -0.018 & 0.0590 & 0.982 & 0.964 & 1.001 \\
\hline hsa-miR-612 & 0.284 & 0.0242 & 1.329 & 1.038 & 1.702 \\
\hline Abbreviations: Cl, confidence interval ; HR, hazard ratio; PTC, papillary thyroid cancer. \\
\hline
\end{tabular}

In the next, we conducted time-dependent ROC curve analysis to assess the performance of our four miRNAs signature in predicting PTC prognosis. The AUC values of the four miRNAs signature at five years were 0.937 and 0.812 in the training cohort and testing cohort, respectively (Fig. 3B). To make the prognostic miRNAs signature more convenient in clinical practice, these four-miRNAs based nomogram was established (Fig. 4A). Furthermore, calibration plots of these four miRNAs based prognostic model showed well compactness in the training cohort and testing cohort at 3-year survival and 5-year survival, respectively, which indicated good calibration ability(Fig. 4B and 4C).

The four miRNAs signature as an independent prognostic factor of OS

The predictive effect of the four miRNAs signature on OS in consideration of clinicopathological features were evaluated adopting the univariate analysis and multivariate Cox regression analysis. Multivariate Cox regression analysis showed that the four miRNAs signature was an independent prognostic factor associated with the OS of PTC patients (Table 4). 
Table 4

Univariate and multivariate cox regression analyses of the four miRNA signature and clinicopathologic factors in the entire set

\begin{tabular}{|c|c|c|c|c|}
\hline & \multicolumn{2}{|c|}{ Univariate analysis } & \multicolumn{2}{|c|}{ Multivariate analysis } \\
\hline & $\mathrm{HR}(95 \% \mathrm{Cl})$ & $P$ & $\mathrm{HR}(95 \% \mathrm{Cl})$ & $P$ \\
\hline Age $(<45$ vs $\geq 45)$ & $\begin{array}{l}0.013(0.000- \\
0.756)\end{array}$ & 0.036 & - & - \\
\hline 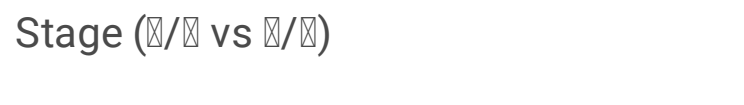 & $\begin{array}{l}0.141(0.045- \\
0.439)\end{array}$ & 0.001 & - & - \\
\hline Sex & $\begin{array}{l}2.029(0.734- \\
5.613)\end{array}$ & 0.173 & - & - \\
\hline $\begin{array}{l}\text { Four miRNA signature (high risk vs low } \\
\text { risk) }\end{array}$ & $\begin{array}{l}8.625(1.956- \\
38.03)\end{array}$ & 0.004 & $\begin{array}{l}6.186(1.405- \\
27.24)\end{array}$ & 0.016 \\
\hline
\end{tabular}

Target prediction and function analysis

The target genes of the selected four miRNAs, hsa-miR-181a-2-3p, hsa-miR-138-5p, hsa-miR-424-3p, and hsa-miR-612, were predicted by TargetScan and miRDB. Then, the biological roles of the overlapped target genes were assessed by the enrichment analysis. The $\mathrm{GO}$ biological processes were mainly enriched in the regulation of nucleobase, nucleoside, nucleotide, and nucleic acid metabolism, transport, regulation of gene expression, epigenetic, and cell adhesion (Table 5). In addition, the KEGG pathways were remarkably enriched in the integrin family cell surface interactions, beta 1 integrin cell surface interactions, proteoglycan syndecan-mediated signaling events (Table 6).

Table 5

The significant enriched GO biological processes of target genes

\begin{tabular}{|llll|}
\hline Term & Count & $\begin{array}{l}\text { Fold } \\
\text { enrichment }\end{array}$ & $\begin{array}{l}\boldsymbol{P} \text { value } \\
\text { value }\end{array}$ \\
\hline $\begin{array}{l}\text { Regulation of nucleobase, nucleoside, nucleotide and nucleic acid } \\
\text { metabolism }\end{array}$ & 291 & 1.228 & 0.001 \\
\hline Transport & 127 & 1.247 & 0.005 \\
\hline Regulation of gene expression, epigenetic & 12 & 2.171 & 0.008 \\
\hline Cell adhesion & 12 & 2.653 & 0.001 \\
\hline
\end{tabular}


Table 6

The significant enriched KEGG pathways of target genes

\begin{tabular}{|llll|}
\hline Term & Count & Fold enrichment & $\boldsymbol{P}$ value \\
\hline Integrin family cell surface interactions & 149 & 1.265 & $<0.001$ \\
\hline Beta1 integrin cell surface interactions & 147 & 1.273 & $<0.001$ \\
\hline Proteoglycan syndecan-mediated signaling events & 146 & 1.2670 & $<0.001$ \\
\hline PAR1-mediated thrombin signaling events & 145 & 1.306 & $<0.001$ \\
\hline Thrombin/protease-activated receptor (PAR) pathway & 145 & 1.305 & $<0.001$ \\
\hline VEGF and VEGFR signaling network & 145 & 1.301 & $<0.001$ \\
\hline Alpha9 beta1 integrin signaling events & 145 & 1.300 & $<0.001$ \\
\hline ErbB receptor signaling network & 145 & 1.294 & $<0.001$ \\
\hline Sphingosine 1-phosphate (S1P) pathway & 145 & 1.294 & $<0.001$ \\
\hline TRAlL signaling pathway & 145 & 1.277 & $<0.001$ \\
\hline
\end{tabular}

\section{Discussion}

In the era of precision medicine, a molecular biomarker-guided treatment and more accurate patient survival prediction are demanding a prompt solution. These efforts have been enormously successful in the field of PTC. With the more often use of thyroid fine-needle aspiration cytology, classification of indeterminate nodules becomes more accurate. However, there is still about $20 \%$ of nodules lack specific characteristics in cytology. ${ }^{14}$. ThyroSeq $v 3$ Genomic Classifier is a tool that includes a variety of thyroid cancer-related point mutations, gene fusions, copy number variations, and gene expression alterations aiming at achieving both high sensitivity and specificity in differentiating benign and malignant thyroid nodules sampled by FNA biopsy ${ }^{15}$. According to the latest research report, the ThyroSeq v3 demonstrated a $94 \%$ sensitivity and $82 \%$ specificity of thyroid nodules with Bethesda III and IV cytology ${ }^{16}$. Besides, BRAF V600E mutation was reported in many studies associated with poorer clinicopathological outcomes of PTC ${ }^{17}$. Our previous studies also showed that co-existence of BRAF V600E and TERT promoter mutations was particularly related to high-risk clinicopathological features of PTC ${ }^{18}$.

With the advancement of sequencing technology and bioinformatics, we can obtain comprehensive DNA epigenetics, mRNA expression profiles, noncoding RNAs, and proteomics data. In the future, a more molecular biomarker-based approach will be developed based on different molecular data. MiRNAs are relatively stable and easily detected. Thus they are promising biomarkers in the clinic. ${ }^{19-21}$. In 2018, Tong Liu et al. constructed a two miRNAs (hsa-miR-181a-2-3p and hsa-miR-138-1-3p) signature for PTC prognosis assessment. The AUC values of the two miRNAs signature were $0.784^{22}$. Also, Xiong Chengfeng et al. also constructed another four miRNAs signature (hsa-mir-6843, hsa-mir-6730, hsa-mir- 
196a-2, and hsa-mir-206) as potential prognostic biomarkers in PTC ${ }^{23}$. The AUC values of the four miRNAs' signature were 0.886 and 0.882 in the training cohort and testing cohort, respectively. In this study, we analyzed large-scale miRNA sequencing data in the TCGA database and selected a total of 2203 miRNAs to provide a more comprehensive analysis. Consequently, we confirmed a four miRNAs signature that has strong prognostic effect on the prognosis of PTC with relatively higher credibility.

It is generally accepted that miRNAs are key regulators of gene expression in complex cellular processes, including cancer cell proliferation, metastasis, migration, and apoptosis. ${ }^{24}$. These four miRNAs found in this study may be potential oncogene or tumor suppressor gene in PTC for its an independent predictor of PTC prognosis. Among these four miRNAs, hsa-miR-181a-2-3p has been reported as a potential prognostic indicator of PTC, its specific function and molecular mechanism in PTC have not been reported $^{22}$. Tong Liu et al. constructed a two-miRNA signature (hsa-miR-181a-2-3p and hsa-miR-138-1-3p) as prognostic biomarkers of PTC prognosis with the AUC was 0.784 .

However, the biological functions of the remaining miRNAs remain unclear. Qingfang $\mathrm{He}$ et al. analyzed the miRNA expression of peripheral blood between healthy people and lung cancer patients, found a significant difference in hsa-miR-138-5p expression in two groups ${ }^{25}$. Besides, previous studies have shown that hsa-miR-138-5p was able to aid early diagnosis of lung adenocarcinoma by blood test in lung cancer presenting with pulmonary nodules ${ }^{26}$. Guanghui Ying et al. also identified hsa-miR-138-5p was significantly down-regulated in renal carcinoma which means hsa-miR-138-5p may contribute to kidney carcinoma development ${ }^{27}$. Hsa-miR-424-3p may be a key miRNA for liver cancer, which was involved in telomere maintenance via telomerase, protein sumoylation, histone mRNA metabolic process, and angiotensin maturation ${ }^{28}$. Chu Tian Xiao et al. identified differences in miRNA expression profiles in peripheral blood exosomes between renal cell carcinoma patients and healthy subjects. They investigated that hsa-mir-424-3p was significantly upregulated in the plasma exosomes of renal cell carcinoma patients compared with healthy controls. For diagnosis of renal cell carcinoma, the AUC of hsa-mir-424-3p was 0.7727 , with the sensitivity of 0.750 , and the specificity of 0.818 , respectively, at the best cutoff value ${ }^{29}$. On the other hand, many studies suggest that the down-regulation of has-miR-612 was involved in a variety of signal pathways that affect the pathophysiology in multiple tumors. Previous studies had shown that miR-612 suppressed the stemness of hepatocellular carcinoma cells by modulating Sp1/Nanog signaling and Wnt/ $\beta$-catenin signaling ${ }^{30,31}$. Study also suggested a critical role of miR-612 in the development of colorectal cancer via restraining cancer cell growth and metastasis ${ }^{32}$. By targeting espin, miR-612 also restrained the growth, invasion, and tumorigenesis of melanoma cells ${ }^{33}$. However, its relationship with PTC remains unclear ${ }^{33-35}$. As a result, it is necessary to conduct additional vitro and in vivo experiments investigations regarding these four miRNAs.

\section{Conclusions}

In conclusion, this study indicated that our four miRNAs signature could accurately predict the prognosis of PTC. For high-risk PTC patients, more intensive treatment and closer follow-up are still recommended. 


\section{Abbreviations}

papillary thyroid cancer, PTC

follicular thyroid cancer, FTC

overall survival, OS

MicroRNAs, miRNAs

The Cancer Genome Atlas, TCGA

fold change, FC

false discovery rate, FDR

Least absolute shrinkage and selection operator analysis, LASSO

the area under the curve, AUC

receiver operating characteristic, ROC

Gene Ontology, GO

Kyoto Encyclopedia of Genes and Genomes, KEGG

\section{Declarations}

Ethics approval and consent to participate:Not applicable.

Consent for publication: Not applicable.

Availability of data and materials: Not applicable.

Competing interests: Not applicable

Funding: This study was funded by the Zhejiang Province Natural Science Foundation of China (No. LGF18H160031) and the Foundation of Wenzhou Municipal Science and Technology Bureau, China (No.Y20170740).

Authors' contributions: Fan Yang completed data analysis and Hongzhong Zhou is responsible for article writing.

Acknowledgements: Not applicable.

\section{References}


1. Siegel RL, Miller KD and Jemal A. Cancer statistics, 2018. CA Cancer J Clin 2018; 68: 7-30. 2018/01/10. DOI: 10.3322/caac.21442.

2. Kilfoy BA, Zheng T, Holford TR, et al. International patterns and trends in thyroid cancer incidence, 1973-2002. Cancer causes \& control : CCC 2009; 20: 525-531. 2008/11/20. DOI: 10.1007/s10552008-9260-4.

3. Siegel RL, Miller KD and Jemal A. Cancer Statistics, 2017. CA Cancer J Clin 2017; 67: 7-30. 2017/01/06. DOI: 10.3322/caac.21387.

4. Wiltshire JJ, Drake TM, Uttley L, et al. Systematic Review of Trends in the Incidence Rates of Thyroid Cancer. Thyroid : official journal of the American Thyroid Association 2016; 26: 1541-1552. 2016/11/02. DOI: 10.1089/thy.2016.0100.

5. McHenry CR and Stulberg JJ. Prophylactic central compartment neck dissection for papillary thyroid cancer. The Surgical clinics of North America 2014; 94: 529-540. 2014/05/27. DOI: 10.1016/j.suc.2014.02.003.

6. Morris LG, Sikora AG, Tosteson TD, et al. The increasing incidence of thyroid cancer: the influence of access to care. Thyroid : official journal of the American Thyroid Association 2013; 23: 885-891. 2013/03/23. DOI: 10.1089/thy.2013.0045.

7. Lewis BP, Burge CB and Bartel DP. Conserved seed pairing, often flanked by adenosines, indicates that thousands of human genes are microRNA targets. Cell 2005; 120: 15-20. 2005/01/18. DOI: 10.1016/j.cell.2004.12.035.

8. Lee JC, Gundara JS, Glover A, et al. MicroRNA expression profiles in the management of papillary thyroid cancer. The oncologist 2014; 19: 1141-1147. 2014/10/18. DOI: 10.1634/theoncologist.20140135.

9. Aragon Han P, Weng $\mathrm{CH}$, Khawaja $\mathrm{HT}$, et al. MicroRNA Expression and Association with Clinicopathologic Features in Papillary Thyroid Cancer: A Systematic Review. Thyroid : official journal of the American Thyroid Association 2015; 25: 1322-1329. 2015/09/29. DOI: 10.1089/thy.2015.0193.

10. Mishra S, Yadav T and Rani V. Exploring miRNA based approaches in cancer diagnostics and therapeutics. Critical reviews in oncology/hematology 2016; 98: 12-23. 2015/10/21. DOI: 10.1016/j.critrevonc.2015.10.003.

11. Sanchez-Vega F, Mina M, Armenia J, et al. Oncogenic Signaling Pathways in The Cancer Genome Atlas. Cell 2018; 173: 321-337.e310. 2018/04/07. DOI: 10.1016/j.cell.2018.03.035.

12. Robinson MD, McCarthy DJ and Smyth GK. edgeR: a Bioconductor package for differential expression analysis of digital gene expression data. Bioinformatics (Oxford, England) 2010; 26: 139140. 2009/11/17. DOI: 10.1093/bioinformatics/btp616.

13. Pathan M, Keerthikumar S, Ang CS, et al. FunRich: An open access standalone functional enrichment and interaction network analysis tool. Proteomics 2015; 15: 2597-2601. 2015/04/30. DOI: 10.1002/pmic.201400515. 
14. Bongiovanni M, Spitale A, Faquin WC, et al. The Bethesda System for Reporting Thyroid Cytopathology: a meta-analysis. Acta cytologica 2012; 56: 333-339. 2012/08/01. DOI: 10.1159/000339959.

15. Nikiforova MN, Mercurio S, Wald Al, et al. Analytical performance of the ThyroSeq v3 genomic classifier for cancer diagnosis in thyroid nodules. Cancer 2018; 124: 1682-1690. 2018/01/19. DOI: 10.1002/cncr.31245.

16. Steward DL, Carty SE, Sippel RS, et al. Performance of a Multigene Genomic Classifier in Thyroid Nodules With Indeterminate Cytology: A Prospective Blinded Multicenter Study. JAMA oncology 2019; 5: 204-212. 2018/11/13. DOI: 10.1001/jamaoncol.2018.4616.

17. Xing $\mathrm{M}$, Westra $\mathrm{WH}$, Tufano RP, et al. BRAF mutation predicts a poorer clinical prognosis for papillary thyroid cancer. The Journal of clinical endocrinology and metabolism 2005; 90: 6373-6379. 2005/09/22. DOI: 10.1210/jc.2005-0987.

18. Jin L, Chen E, Dong S, et al. BRAF and TERT promoter mutations in the aggressiveness of papillary thyroid carcinoma: a study of 653 patients. Oncotarget 2016; 7: 18346-18355. 2016/03/05. DOI: 10.18632/oncotarget.7811.

19. Qiu J, Zhang W, Zang C, et al. Identification of key genes and miRNAs markers of papillary thyroid cancer. Biological research 2018; 51: 45. 2018/11/12. DOI: 10.1186/s40659-018-0188-1.

20. Mazeh H, Deutch T, Karas A, et al. Next-Generation Sequencing Identifies a Highly Accurate miRNA Panel That Distinguishes Well-Differentiated Thyroid Cancer from Benign Thyroid Nodules. Cancer epidemiology, biomarkers \& prevention : a publication of the American Association for Cancer Research, cosponsored by the American Society of Preventive Oncology 2018; 27: 858-863. 2018/07/28. DOI: 10.1158/1055-9965.Epi-18-0055.

21. Nixon AM, Provatopoulou X, Kalogera E, et al. Circulating thyroid cancer biomarkers: Current limitations and future prospects. Clinical endocrinology 2017; 87: 117-126. 2017/05/12. DOI: 10.1111/cen.13369.

22. Liu T, You X, Sui J, et al. Prognostic value of a two-microRNA signature for papillary thyroid cancer and a bioinformatic analysis of their possible functions. Journal of cellular biochemistry 2018 2018/11/06. DOI: 10.1002/jcb.27993.

23. Chengfeng X, Gengming C, Junjia Z, et al. MicroRNA signature predicts survival in papillary thyroid carcinoma. Journal of cellular biochemistry 2019; 120: 17050-17058. 2019/05/18. DOl: 10.1002/jcb.28966.

24. Hill M and Tran N. MicroRNAs Regulating MicroRNAs in Cancer. Trends in cancer 2018; 4: 465-468. 2018/06/26. DOI: 10.1016/j.trecan.2018.05.002.

25. He Q, Fang Y, Lu F, et al. Analysis of differential expression profile of miRNA in peripheral blood of patients with lung cancer. Journal of clinical laboratory analysis 2019; 33: e23003. 2019/09/22. DOI: 10.1002/jcla.23003.

26. He Y, Ren $S$, Wang $Y$, et al. Serum microRNAs improving the diagnostic accuracy in lung cancer presenting with pulmonary nodules. Journal of thoracic disease 2018; 10: 5080-5085. 2018/09/21. 
DOI: $10.21037 /$ jtd.2018.07.138.

27. Ying $G, W u R$, Xia $M$, et al. Identification of eight key miRNAs associated with renal cell carcinoma: $A$ meta-analysis. Oncology letters 2018; 16: 5847-5855. 2018/10/23. DOI: 10.3892/ol.2018.9384.

28. Wang X, Gao J, Zhou B, et al. Identification of prognostic markers for hepatocellular carcinoma based on miRNA expression profiles. Life sciences 2019; 232: 116596. 2019/06/25. DOI: 10.1016/j.Ifs.2019.116596.

29. Xiao CT, Lai WJ, Zhu WA, et al. MicroRNA Derived from Circulating Exosomes as Noninvasive Biomarkers for Diagnosing Renal Cell Carcinoma. Onco Targets Ther 2020; 13: 10765-10774. 2020/10/31. DOI: 10.2147/ott.S271606.

30. Tang J, Tao ZH, Wen D, et al. MiR-612 suppresses the stemness of liver cancer via Wnt/ $\beta$-catenin signaling. Biochemical and biophysical research communications 2014; 447: 210-215. 2014/04/08. DOI: 10.1016/j.bbrc.2014.03.135.

31. Liu Y, Liu DL, Dong LL, et al. miR-612 suppresses stem cell-like property of hepatocellular carcinoma cells by modulating Sp1/Nanog signaling. Cell death \& disease 2016; 7: e2377. 2016/09/30. DOI: 10.1038/cddis.2016.282.

32. Sheng $L, H e P$, Yang $X$, et al. miR-612 negatively regulates colorectal cancer growth and metastasis by targeting AKT2. Cell death \& disease 2015; 6: e1808. 2015/07/15. DOI: 10.1038/cddis.2015.184.

33. Zhu Y, Zhang HL, Wang QY, et al. Overexpression of microRNA-612 Restrains the Growth, Invasion, and Tumorigenesis of Melanoma Cells by Targeting Espin. Molecules and cells 2018; 41: 119-126. 2018/02/02. DOI: 10.14348/molcells.2018.2235.

34. Yu H, Xu Y, Zhang D, et al. Long noncoding RNA LUCAT1 promotes malignancy of ovarian cancer through regulation of miR-612/HOXA13 pathway. Biochemical and biophysical research communications 2018; 503: 2095-2100. 2018/08/16. DOI: 10.1016/j.bbrc.2018.07.165.

35. Wang $L$, Bo X, Zheng Q, et al. Paired box 8 suppresses tumor angiogenesis and metastasis in gastric cancer through repression of FOXM1 via induction of microRNA-612. Journal of experimental \& clinical cancer research : CR 2018; 37: 159. 2018/07/20. DOI: 10.1186/s13046-018-0830-3.

\section{Figures}




\section{Volcano}

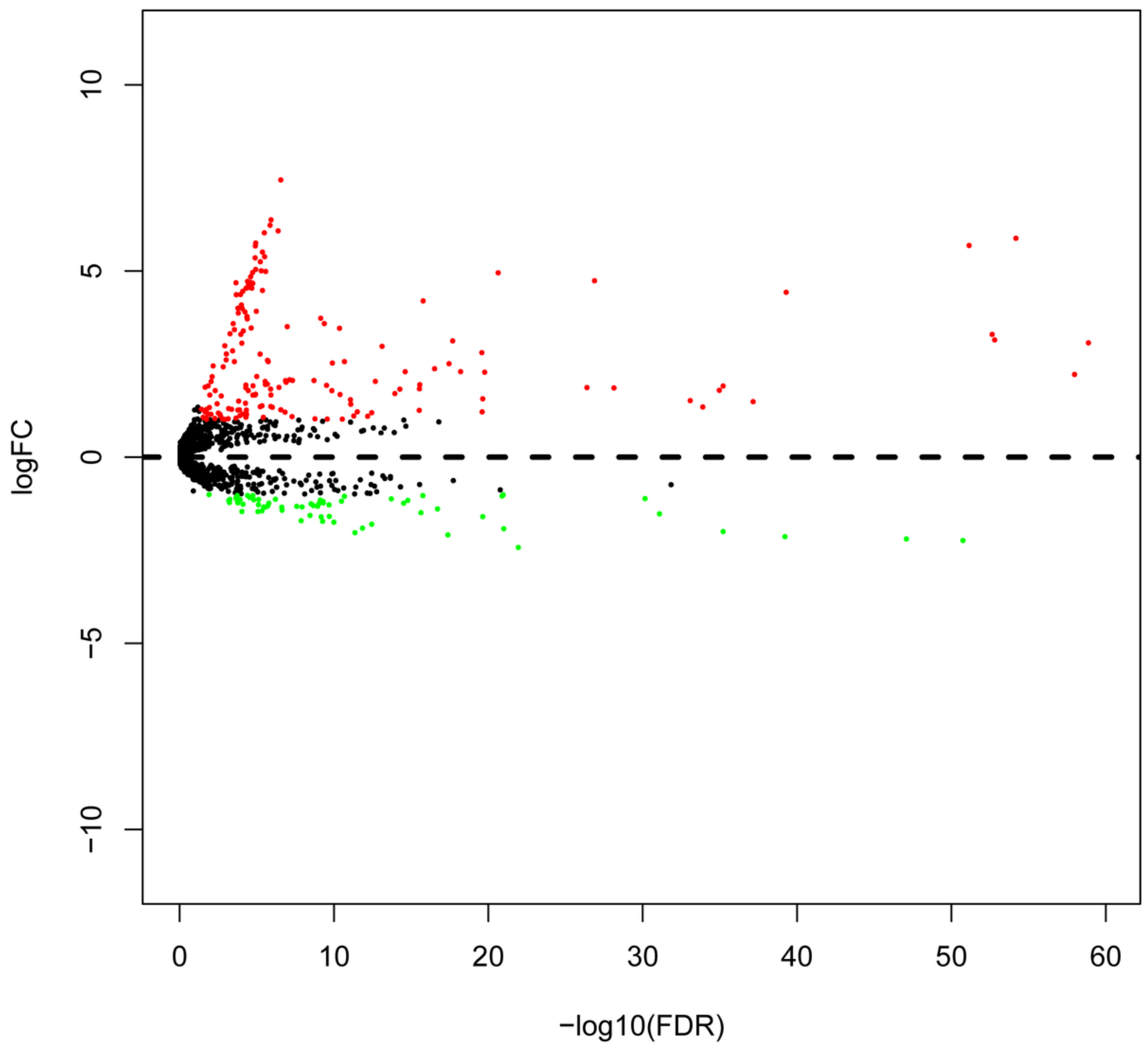

Figure 1

Volcano plot of 2202 miRNAs in PTC patients. Red color indicates up-regulated expression, and green color represents down-regulated expression. Abbreviations: FDR, false discovery rate; FC, fold change. 
A

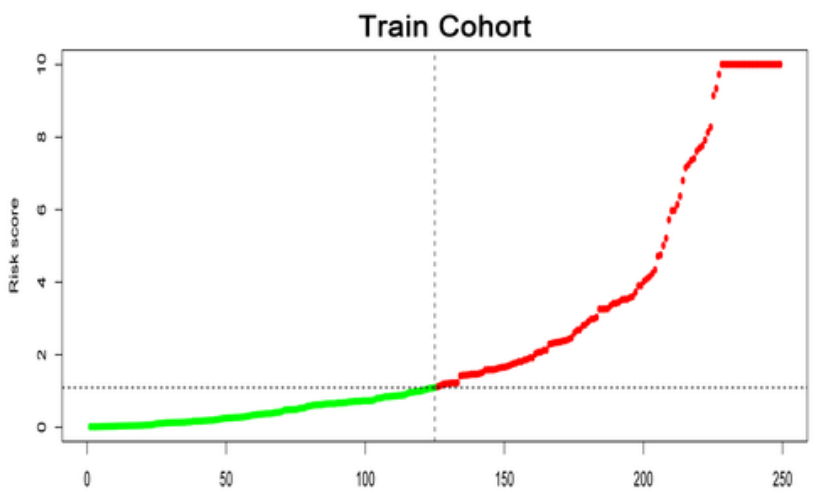

B

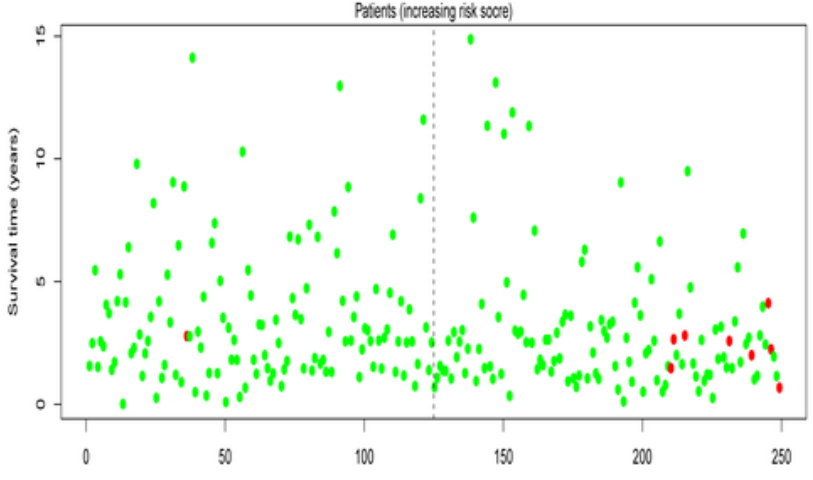

C

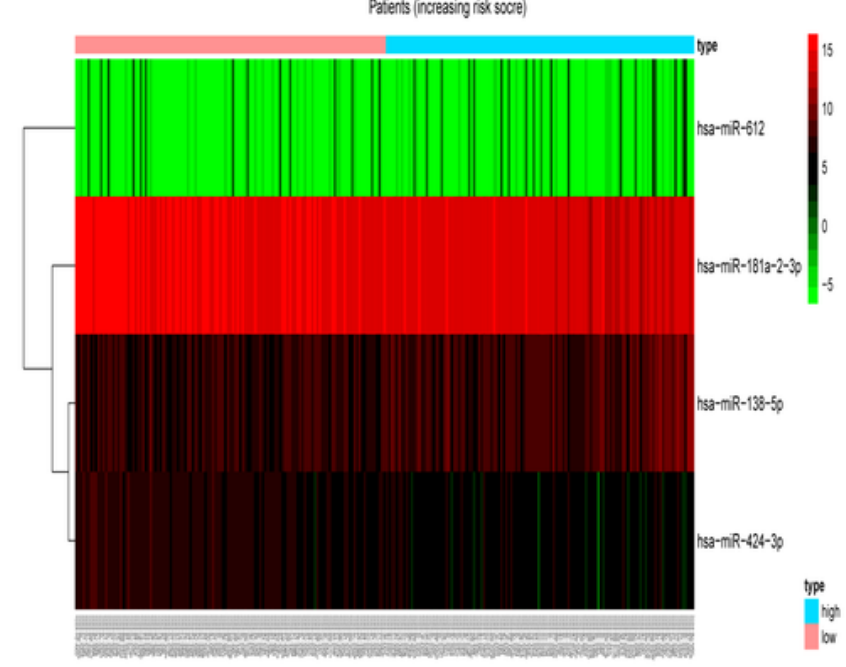

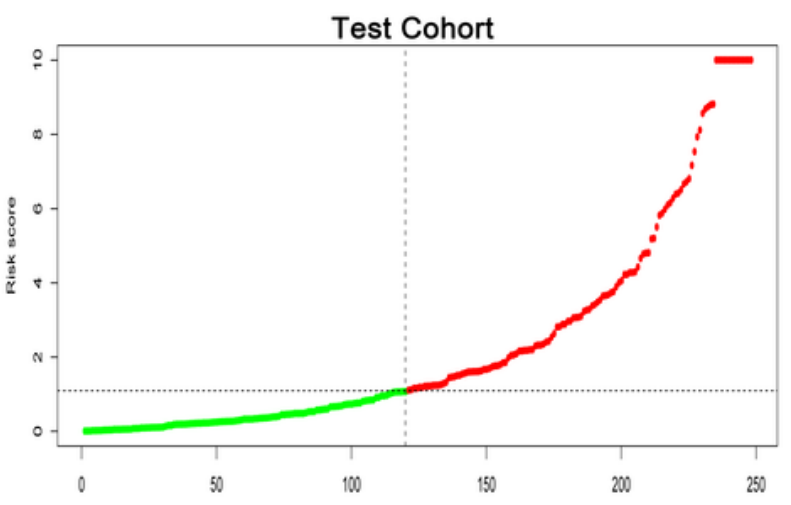

Pajents increasing risk socre

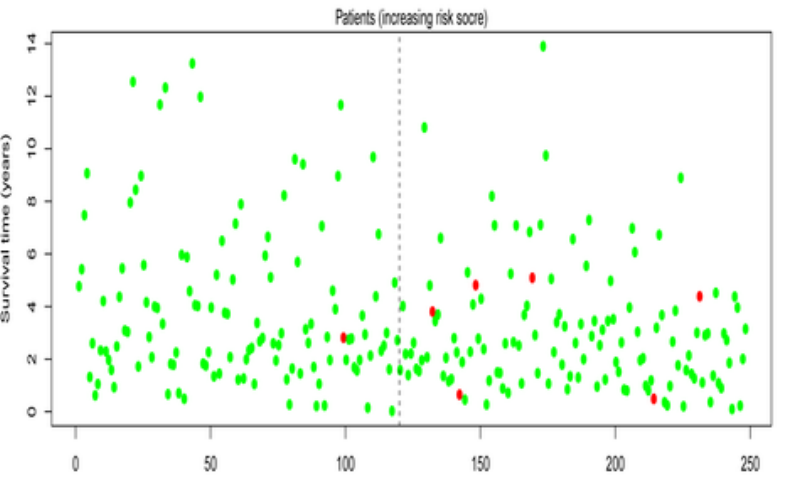

Pasents (noressingisisk sore)

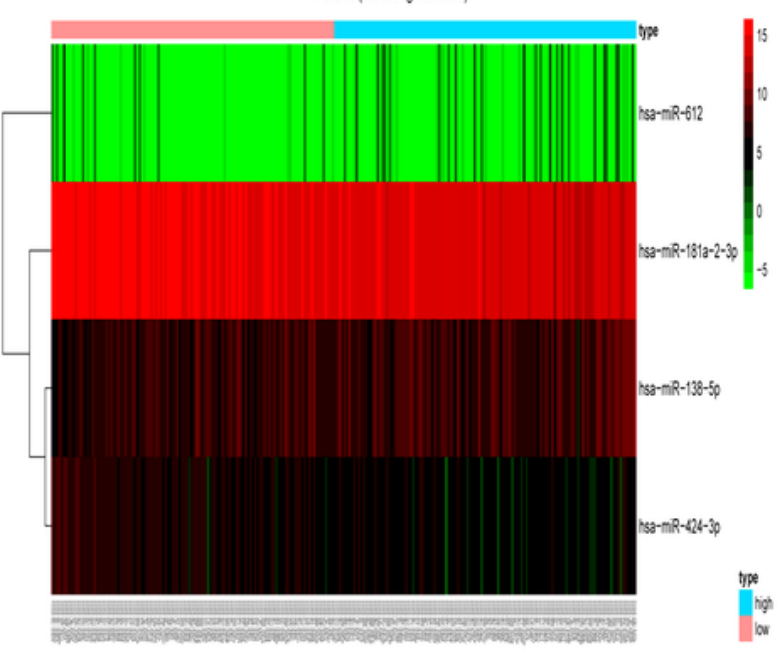

\section{Figure 2}

The distribution of risk score, OS, and OS status and the heat map of prognostic four miRNA signature in (A) Train cohort and (B) Tset cohort. The dotted line indicates the cutoff point of the median risk score used to stratify patients into the low-risk group and high-risk group. 
A

Train Cohort

Survival curve $(p=7.932 e-03)$

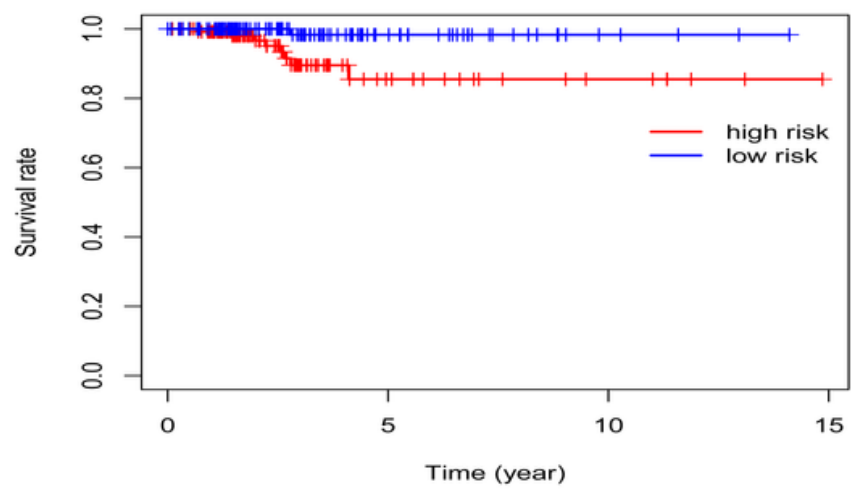

B

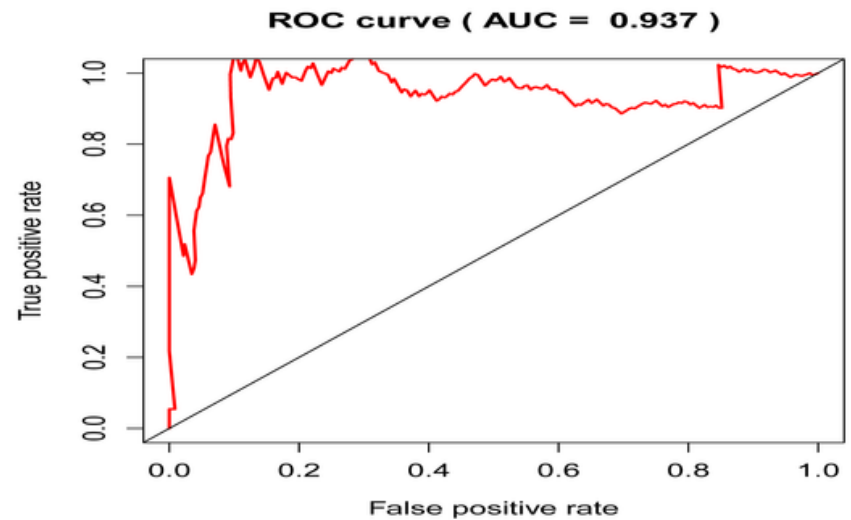

Test Cohort

Survival curve $(p=2.728 e-02)$

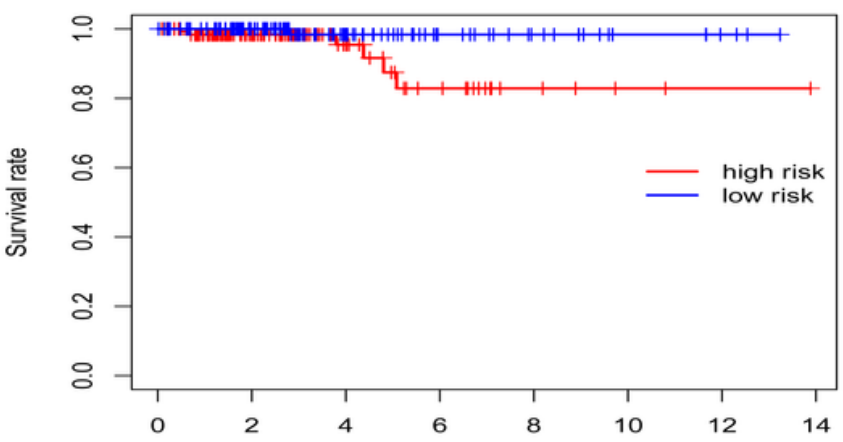

ROC curve ( $A U C=0.812$ )

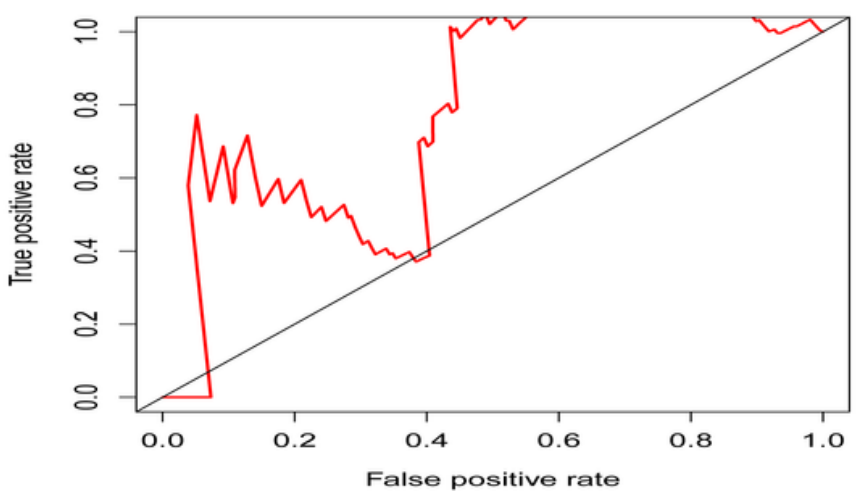

\section{Figure 3}

Kaplan-Meier curves of overall survival and time-dependent ROC curves of 5-year survival for PTC patients based on the four miRNA signature in the (A) Train cohort and (B) Tset cohort. 
A

Points

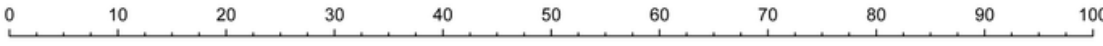

hsa-miR-181a-2-3p

hsa-miR-138-5p

hsa-miR-424-3p

hsa-miR-612

Total Points

3-Year Survival

5-Year survival

B

C
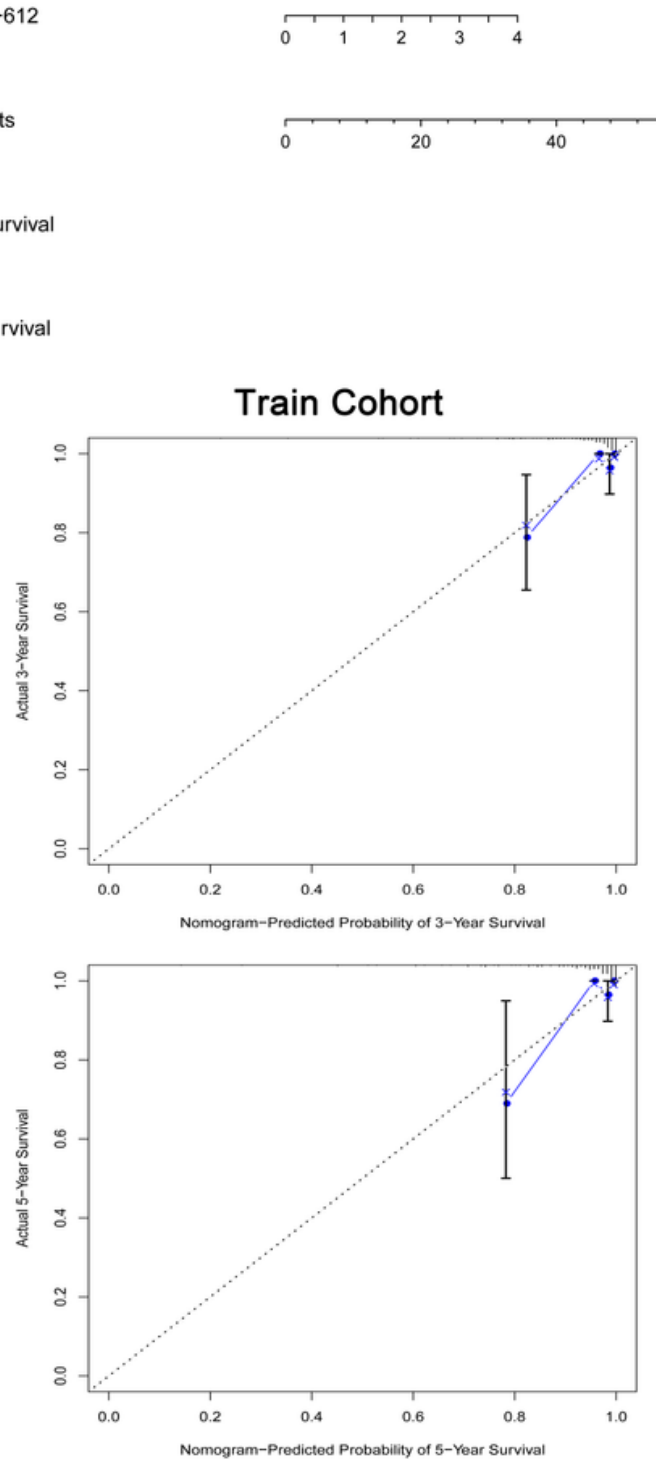
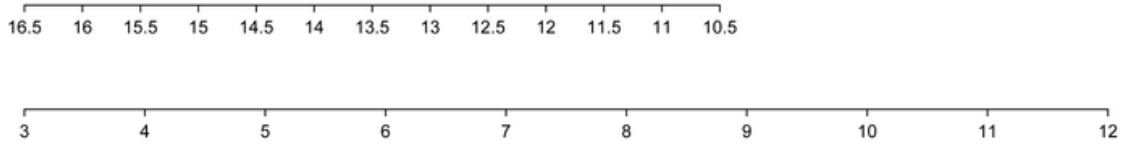

$\begin{array}{lllllllllllllllll}8.5 & 8 & 7.5 & 7 & 6.5 & 6 & 5.5 & 5 & 4.5 & 4 & 3.5 & 3 & 2.5 & 2 & 1.5 & 1\end{array}$

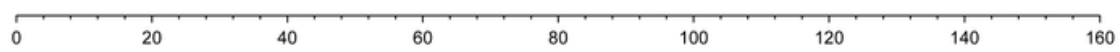

\begin{tabular}{lllllll}
\hline 0.9 & 0.8 & 0.7 & 0.6 & 0.50 .40 .30 .2 & 0.1
\end{tabular}

\begin{tabular}{lllllll}
\hline 0.9 & 0.8 & 0.7 & 0.6 & 0.50 .40 .30 .2 & 0.1
\end{tabular}

Test Cohort
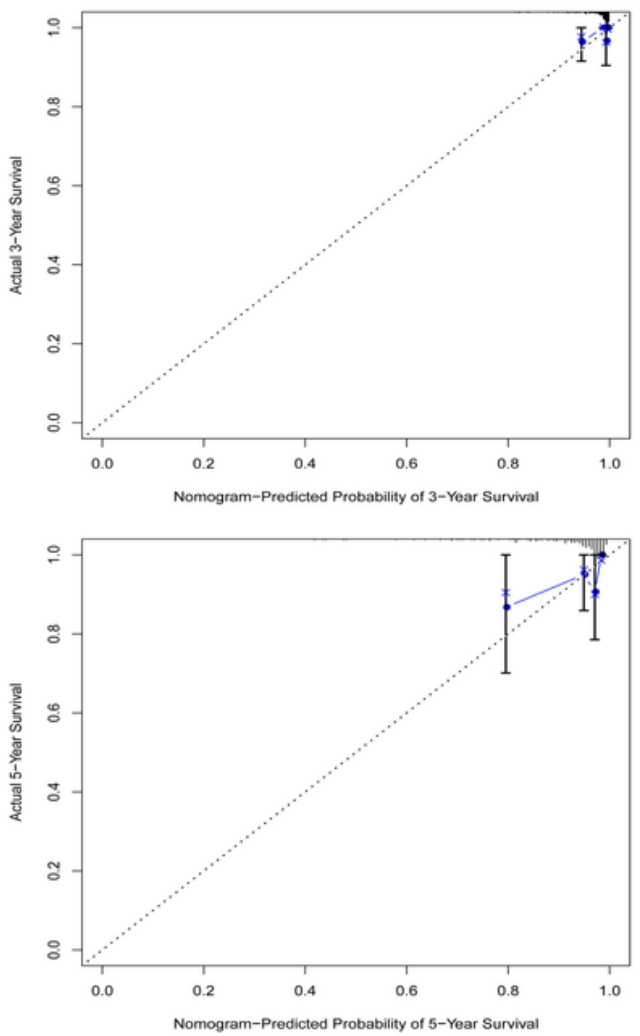

Figure 4

(A) The prognostic nomogram based on four miRNA signature. (B) calibration plots of the four miRNA based prognostic model in the train cohort and tset cohort of 3-year survival; (C) calibration plots of the four miRNAs based prognostic model in the train cohort and tset cohort of 5-year survival.

\section{Supplementary Files}


This is a list of supplementary files associated with this preprint. Click to download.

- supplementaryfigure1.tif

- supplementaryfigure2.tif

- SupplementaryTable1.xlsx

- SupplementaryTable2.xIsx 